\title{
Severe Impaired Bone Formation was Induced by Short-Term Fasting in Adult Mice
}

\author{
Jiajing Chen ${ }^{1}$, Rui He ${ }^{1}$, Juan $\mathrm{Li}^{2}$, Yan Zhang ${ }^{3}$, Jinwei Qi ${ }^{4}$, Xianghui Meng ${ }^{5}$, Yuying Wang ${ }^{1, *}$ \\ ${ }^{1}$ College of Life and Environmental Sciences, Hangzhou Normal University, Hangzhou, China \\ ${ }^{2}$ Department of Reproduction and Genetics, Hebei General Hospital, Shijiazhuang, China \\ ${ }^{3}$ Changzhan Hospital of Shijiazhuang, Shijiazhuang, China \\ ${ }^{4}$ Department of Urology, the Hospital of Lingshou, Shijiazhuang, China \\ ${ }^{5}$ Jizhou District Hospital of Hengshui, Hengshui, China

\section{Email address:} \\ 531683700@qq.com (Jiajing Chen),41499031@qq.com (Rui He), liwang0603@163.com (Juan Li), 4978465@qq.com (Yan Zhang), \\ 1369825915@qq.com (Jinwei Qi), mengxh120@126.com (Xianghui Meng), 1918114030@qq.com (Yuying Wang) \\ *Corresponding author
}

\section{To cite this article:}

Jiajing Chen, Rui He, Juan Li, Yan Zhang, Jinwei Qi, Xianghui Meng, Yuying Wang. Severe Impaired Bone Formation was Induced by Short-Term Fasting in Adult Mice. American Journal of Clinical and Experimental Medicine. Vol. 4, No. 6, 2016, pp. $212-215$. doi: 10.11648/j.ajcem.20160406.19

Received: November 14, 2016; Accepted: December 5, 2016; Published: December 7, 2016

\begin{abstract}
The alterations in bone metabolism were associated with food and nutrition. Regrettably is that no studies have analyzed the bone microstructure subjected to complete fasting in mammals. Our study aimed to identify the effects and alterations of complete fasting on bones via histomorphometric analysis in adult female mice. Twelve-week-old C57BL/6J female mice were randomly divided into ad libitum (AL) and two-day complete fasting (FA) groups. Histomorphometry was carried out to analyze the differences in the microstructure of femurs after the experiment period. The histomorphometric data indicated that the bone resorption parameters, including the number of osteoclasts (Mu.N.Oc/B.Pm), osteoclast surface (Oc.S/BS), and eroded surface (ES/BS), were similar between the two groups. However, the bone formation parameters, including the number of osteoblasts (N.Ob/B.Pm) and osteoblast surface (Ob.S/BS), were markedly decreased in the FA group compared with the AL group. Thus, bone formation but not bone resorption was severely impaired following complete fasting. Furthermore, the osteoid surface (OS/BS) was observably reduced, which indicates that the bone mass should be reduced. However, there was no dramatic reduction in the osteoid thickness (O.Th); thus, the bone mass remained relatively stable between the two groups. These findings provide new insights in the increased incidence of osteoporosis in individuals on weight-reduction diets and other bone diseases associated with undernutrition.
\end{abstract}

Keywords: Boneformation, Osteoblast, Bone Resorption, Osteoclast, Histomorphometry

\section{Introduction}

Food and nutrition play important roles in the prevention of many diseases. Furthermore, the balanced diet, which can provide essential nutrients, is beneficial to thebody weightand organmass [1]. Chronic undernutrition isassociated with loss in bone mass andalterationin bone metabolism. The increase in osteoporosisincidence and the reduction in bone formation have been associated with the degree of dietary restriction in women [2-6]. Several studies have also demonstrated the effects of short-term complete fasting on bone turnover. About half of reduction in bone formation was identified in 4-day complete fasting women [7]. Bone turnover was also reduced by $24 \mathrm{hr}$ of fasting in male undergraduate students [8]. Similar results have been reported in theresearches about animals. Significant effects of food restriction on bone metabolism have been identified in mice [9-12]. Unfortunately, no previous study about microstructural analysis of bone in animals or humans following complete fasting was implemented. In our study, we conducted a comprehensive microstructural analysis of the following parameters: the trabecularbone volume (bone volume/tissue volume, BV/TV), 
osteoid surface (OS/BS), osteoid thickness (O.Th), number of osteoblasts (N.Ob/B.Pm), osteoblast surface (Ob.S/BS), number of multinucleated osteoclasts (Mu.N.Oc/B.Pm), osteoclast surface (Oc.S/BS), and eroded surface (ES/BS).

Bone histomorphometry is used to analyze a two-dimensional bone tissue section. Parameters of the bone microstructurecan be identified through target analysis. Compared with the measurement technology used to determine bone mineral density, histomorphometry is a superior techniquebecause it can qualitatively identify the morphology of bone tissue. Moreover, it can provide an objective measure of bone biology performance, including the thickness and void ratio of the cortex, the area and thickness of the trabecular bone, and the amount of trabecular bone connection [13-15]. This histomorphometric data can provide critical information regarding the bone microstructure.

Summary, no previous studies have investigated the bone microstructurein fastingmice. In this study, adult female mice were subjected to a 2-day period of complete fasting. The aim of the research was to identify the effects of complete short-term fasting on femur in food-restricted mice via histomorphometric analysis.

\section{Materials and Methods}

\subsection{Animal Model}

Twelve week-old C57BL/6J female mice were housed in the Hangzhou Normal University Animal Center. The mice were provided with food and water ad libitum and maintained on a 12-hour light/dark cycle, under controlled temperature and humidity conditions. Prior to the study, all experiments were reviewed and approved by the Animal Care and Use Committee of Hangzhou Normal University, Zheijiang, China. The mice were randomly divided into two groups $(\mathrm{n}=$ 8/group): the ad libitum (AL) control group and an experimental group that underwent complete fasting with ad libitum water for three days (FA). Two days were chosen based on a pilot experiment that was conducted in our group, which indicated mice could endure extreme fasting conditions for 2 days. At the start of the study, the mice in the FA group were transferred to a new clean cage without access to food at Zeitgeber time 20 (ZT20). These mice were subsequently sacrificed at ZT8 on the second day of fasting [8]. The body weights of the mice were monitored during the experimental period. The left femur of each mouse was harvested, preserved in $70 \%$ ethanol, and subsequently processed for histomorphometric analysis.

\subsection{Histomorphometric Analysis of the Femur}

Each femur was cut into two parts: the proximal femur and the middle/distal femur. The proximal metaphysis was stained with Villanueva Osteochrome Bone Stain (Merck, Darmstadt, Germany) for seven days. The specimens were subsequently dehydrated, defatted sequentially in increasing concentrations of ethanol $(70 \%, 95 \%, 99.5 \%$, and $100 \%)$ and acetone, respectively, and embedded in methyl methacrylate (Wako, Osaka, Japan). Six- $\mu$ m-thick longitudinal sections of the proximal metaphysis were cut on a Leica RM2255microtome (Leica, Inc., Nussloch, Germany). The samples were then transferred onto a chromium/gelatin-coated slide and dried overnight under pressure at $42^{\circ} \mathrm{C}$ and cover slipped with acrylic resins (Maruto, Tokyo, Japan). These sections were analyzed using histomorphometric software (System Supply Co., Nagano, Japan). The nomenclature, symbols, and units have been recommended by the Nomenclature Committee of the American Society for Bone and Mineral Research [16].

\subsection{Statistical Analysis}

The data are expressed as the mean \pm standard deviation (S. D.). Significant differences between the AL and FA groups were identified using Student's t-tests. The effects were considered to be statistically significant when $p<0.05$.

\section{Results}

\subsection{Effect of Fasting on Body Weight}

In the FA group, the mean body weight (starting weight $=$ $19.3 \pm 1.6 \mathrm{~g}$ ) significantly decreased by approximately $20 \%$ following two days of complete fasting and was significantly lower $(15.4 \pm 1.1 \mathrm{~g})$ compared with the AL group $(20.2 \pm 0.9 \mathrm{~g}$; Fig. 1). Although the mice in the FA group experienced substantial weight loss $(\mathrm{p}<0.05)$, no mortality or morbidity occurred during the fasting period.

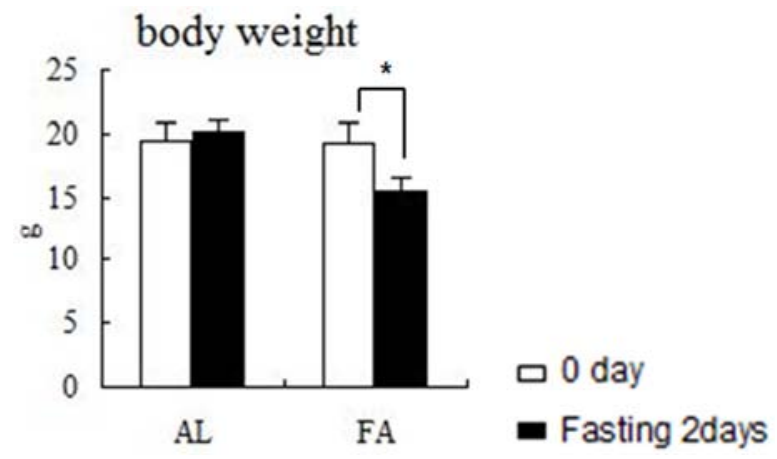

Figure 1. Mean body weight following two-day complete fasting (FA) or ad libitum food (AL) in adult female mice. $n=8$ per group. $* p<0.05$ vs. control.

\subsection{Influence of Fasting on the Trabecular and Cortical Bones}

The histomorphometric data indicated there were no significant group differences in the Mu.N.Oc/B.Pm, Oc.S/BS, ES/BS, BV/TV, or O.Th (Fig. 2e, f, g, h, b). However, the bone formation parameters, including the OS/BS, N.Ob/B.Pm, and Ob.S/BS (Fig. 2a, c, d), were significantly decreased in the femur sections of the FA group. The histomorphometric analysis indicated there were no significant differences in bone resorption between the FA and AL groups; however, there was a significant reduction in bone formation in the FA group compared with AL group ( $p$ $<0.05)$. 
a

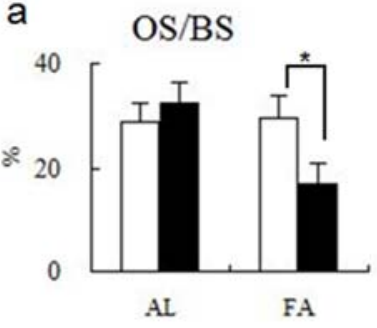

C
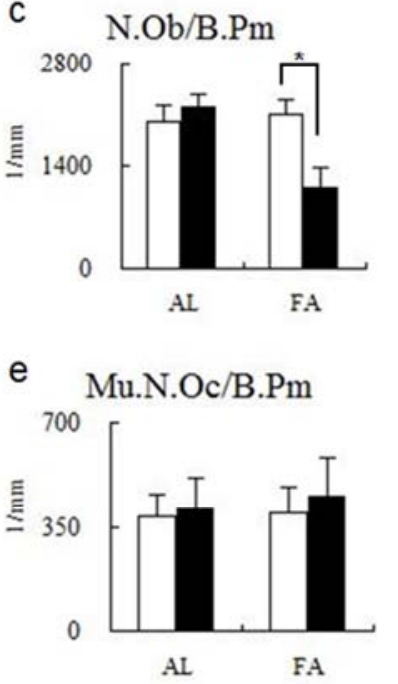

g

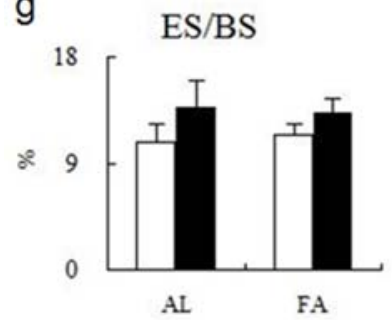

口 0 day

\section{- Fasting 2days}

Figure 2. Bone histomorphometric analyses following two days of complete fasting in adult female mice. The osteoid surface (OS/BS), osteoid thickness (O.Th), number of osteoblasts (N.Ob/B.Pm), osteoblast surface (Ob.S/BS), number of multinucleated osteoclasts (Mu.N.Oc/B.Pm), osteoclast surface $(O c . S / B S)$, and eroded surface (ES/BS), trabecular bone volume (bone volume/tissue volume, $B V / T V$ ) are shown. Data represent the mean \pm standard deviation (SD) offive mice. ${ }^{*} p<0.05$ vs. control.

\section{Discussion}

The purpose of our project was to evaluate the effects of two-day complete fasting on bone turnover via the measurement of microstructure. Weight loss has been associated with bone status $[1,17,18]$. However, previous studies have not reported a quantification of the morphological changes in the bone microstructure. The histomorphometric analysis clearly demonstrated the transformation in the femur, including the invariability in bone resorption and the variability in bone formation. The current findings regarding the detailed analysis of the bone microstructure indicate that during the fasting period, bone loss occurred due to changes in osteoblasts rather than osteoclasts. This finding provides novel insights that may be relevant to individuals with osteoporosis following diets or other chronicmalnourishment diseases.

\section{Acknowledgements}

This work was supported by Natural Science Foundation of Zhejiang Province (LQ13H060003) and National Natural Science Foundation of China (81401811).

\section{References}

[1] Reid IR. "Relationships among body mass, its components, and bone," Bone. vol. 31, no. 5, pp. 547-555, Nov. 2002.

[2] Fazeli PK, Klibanski A. "Anorexia nervosa and bone metabolism,” Bone. vol. 66, pp. 39-45, Sep. 2014.

[3] Goebel G, Schweiger U, Krüger R, et al. "Predictors of bone mineral density in patients with eating disorders," Int $\mathrm{J}$ Eat Disord, vol .25, no. 2, pp. 143-150, Mar. 1999.

[4] Hampson G, Martin FC, Moffat K, et al. "Effects of dietary improvement on bone metabolism in elderly underweight women with osteoporosis: a randomised controlled trial," Osteoporos Int. vol. 14, no. 9, pp. 750-756, Sep. 2003.

[5] Devine A Dick IM, Islam AF, et al. "Protein consumption is an important predictor of lower limb bone mass in elderly women," Am J Clin Nutr. vol. 81, no. 6, pp. 1423-1428, Jun. 2005.

[6] Ihle R, Loucks AB."Dose-response relationships between energy availability and bone turnover in young exercising women," J Bone Miner Res. vol. 19, no. 8, pp. 1231-1240, Aug. 2004.

[7] Grinspoon SK, Baum HB, Kim V, et al."Decreased bone formation and increased mineral dissolution during acute fasting in young women," J Clin Endocrinol Metab, vol. 80, no. 12, pp. 3628-3633, Dec. 1995.

[8] Talbott SM, Shapses SA."Fasting and energy intake influence bone turnover in lightweight male rowers," Int J Sport Nutr, vol. 8, no4, pp. 377-387, Dec. 1998.

[9] Hamrick MW, Ding KH, Ponnala S, et al."Caloric restriction decreases cortical bone mass but spares trabecular bone in the mouse skeleton: implications for the regulation of bone mass by body weight," J Bone Miner Res, vol. 23, no. 6, pp. 870-878, Jun. 2008.

[10] Brochmann EJ, Duarte ME, Zaidi HA, et al."Effects of dietary restriction on total body, femoral, and vertebral bone in SENCAR, C57BL/6, and DBA/2 mice," Metabolism, vol. 52, no. 10 , pp. $1265-1273$, Oct. 2003.

[11] Shushimita S, de Bruijn MJ, de Bruin RW, et al. Dietary restriction and fasting arrest $\mathrm{B}$ and $\mathrm{T}$ cell development and increase mature B and T cell numbers in bonemarrow," PLoS One, vol. 9, no. 2, pp. e87772, Feb. 2014.

[12] Fujita Y, Yanagida H, Mimori T, et al."Prevention of fasting-mediated bone marrow atrophy by leptin administration," Cell Immunol, vol. 273, no. 1, pp. 52-58, 2012. 
[13] Barger-Lux MJ, Recker RR."Bone microstructure in osteoporosis: transilial biopsy and histomorphometry," Top Magn Reson Imaging, vol. 13, no. 5, pp. 297-305, Oct. 2002.

[14] Hauser R, Barres D, Durigon M, et al."Identification using histomorphometry of the femur and tibia," Acta Med Leg Soc (Liege), vol. 30, no. 2, pp. 91-97, 1980.

[15] Nakabayashi Y, Wevers HW, Cooke TD, et al."Bone strength and histomorphometry of the distal femur," J Arthroplasty, vol. 9, no. 3, pp. 307-315, Jun. 1994.

[16] Parfitt AM. "Bone histomorphometry: proposed system for standardization of nomenclature, symbols, and units," Calcif Tissue Int, vol. 42, no. 5, pp. 284-286, May. 1988.

[17] Josse AR, Atkinson SA, Tarnopolsky MA, et al. "Diets higher in dairy foods and dietary protein support bone health during diet- and exercise-induced weight lossin overweight and obese premenopausal women," J Clin Endocrinol Metab, vol. 97, no. 1, pp. 251-260, Jan. 2012.

[18] Nebot E1, Erben RG, Porres JM, et al. "Effects of the amount and source of dietary protein on bone status in rats," Food Funct, vol. 5, no 4, pp. 716-23, Apr. 2014. 